\title{
Electrically Tunable Bandgaps in Bilayer $\mathrm{MoS}_{2}$
}

\section{Supplemental Information}

Tao Chu, Hesameddin Ilatikhameneh, Gerhard Klimeck, Rajib Rahman, Zhihong Chen

School of Electrical and Computer Engineering and Birck Nanotechnology Center, Purdue University, West Lafayette, IN, USA

*Correspondence to: zhchen@purdue.edu

\section{Temperature Dependent Measurements of Bilayer MoS $\underline{S}_{2}$ Devices}

Temperature dependent measurements are implemented for bilayer MoS2 devices with $20 \mathrm{~nm} \mathrm{HfO2}$, as shown in Fig. S1. As T decreases from $270 \mathrm{~K}$ to $77 \mathrm{~K}$, the off-state current decrease and the subthreshold slope of both branch become steeper. For all the temperatures, off-state current increases as the electric field increases. Bandgap size is difficult to extract from the temperature dependent measurements due to the large SB in the p branch, because the SB branch show a different $\mathrm{T}$ dependence compared to the thermal branch. Instead, threshold voltage method is used for bandgap change extraction, as shown in Fig. S2. For different temperatures, the trend of the bandgap change is similar.

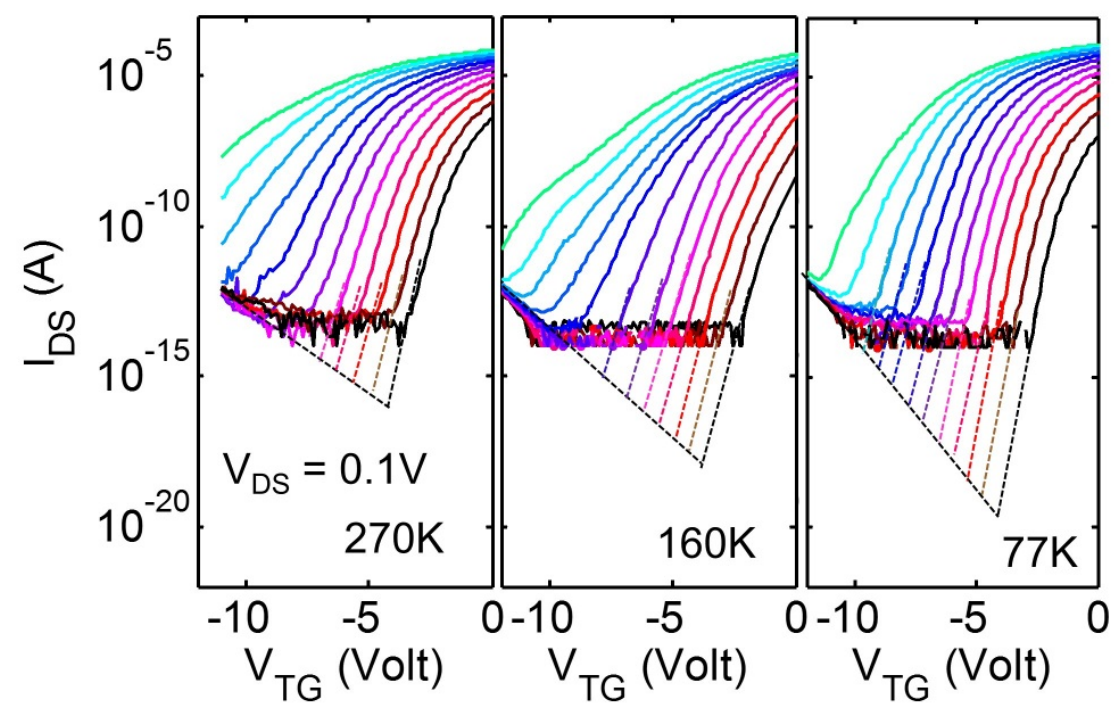

Fig. S1: Temperature dependent measurements for a dual gate bilayer $\mathrm{MoS}_{2}$ FET with a $20 \mathrm{~nm} \mathrm{HfO}_{2}$ gate oxide. Transfer characteristics measured at $\mathrm{T}=270 \mathrm{~K}, 160 \mathrm{~K}$, and $77 \mathrm{~K}$ for $\mathrm{V}_{\mathrm{BG}}=-50 \mathrm{~V}$ to $+60 \mathrm{~V}$ with step of $10 \mathrm{~V}$. Device off-states are obtained by extension of the $\mathrm{n}$ - and $\mathrm{p}$-branches. 


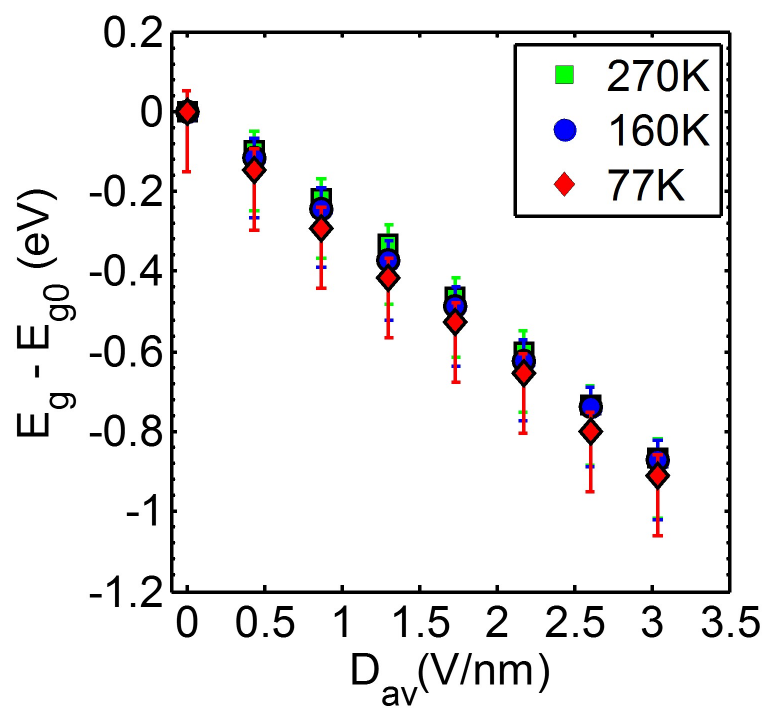

Fig. S2: Extracted bandgap change as a function of $D_{a v}$, based on the threshold voltage method described in the paper. The bandgap change is independent of temperature.

\section{Transport Characteristics of a Bilayer MoS ${ }_{2}$ Device with an HSQ Buffer Layer}

Transfer characteristics of a bilayer $\mathrm{MoS}_{2}$ device with a HSQ buffer layer and $20 \mathrm{~nm} \mathrm{HfO}_{2}$ are shown in Fig. S3. Similar to the device in Fig. S1, the bandgap is decreasing as the electric field increases.
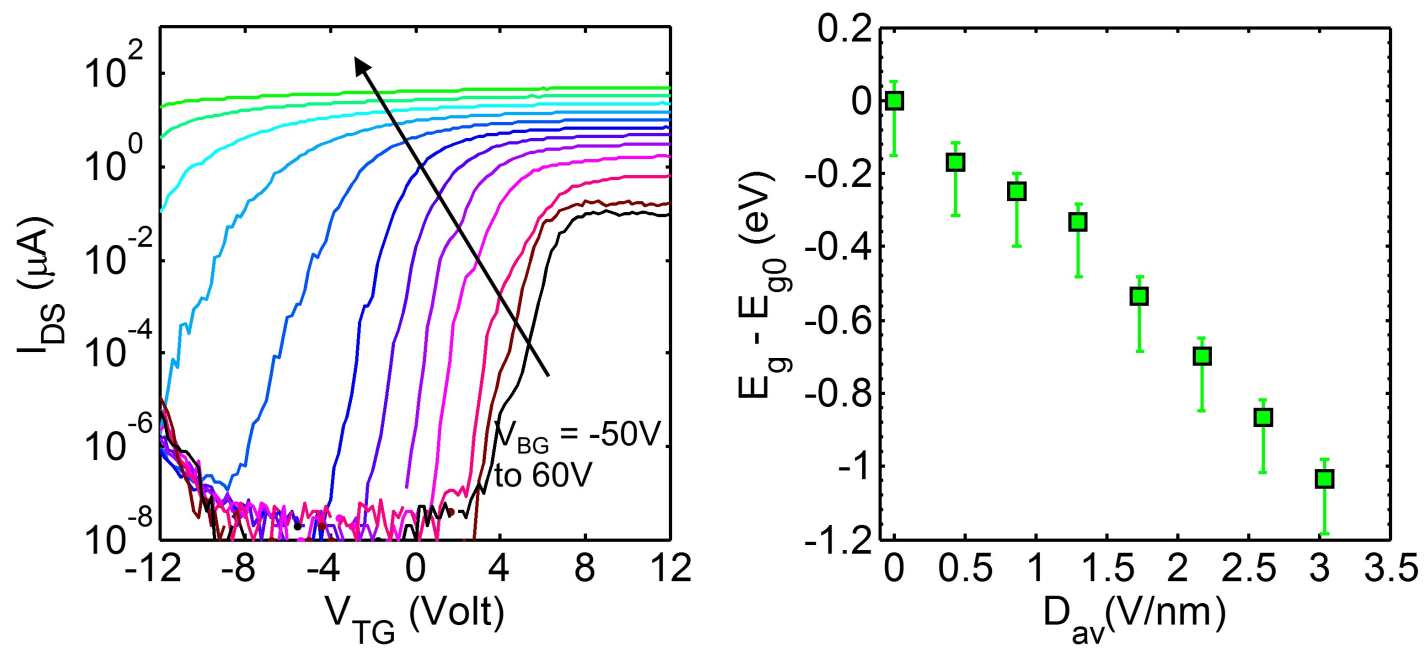

Fig. S3: (a) Transfer characteristics for a dual-gated bilayer $\mathrm{MoS}_{2}$ FET with a HSQ buffer layer and $20 \mathrm{~nm} \mathrm{HfO}_{2}$ gate oxide, measured at $\mathrm{V}_{\mathrm{BG}}=-50 \mathrm{~V}$ to $+60 \mathrm{~V}$ with a step of $10 \mathrm{~V}$. (b) Extracted bandgap change as a function of $\mathrm{D}_{\mathrm{av}} .1 .2 \mathrm{eV}$ bandgap reduction is achieved at $D_{a v}=3 V / n m$. 


\section{Photoluminescence Measurement of Bilayer MoS2 Devices After Each Fabrication Step}

It is non-trivial to make PL measurements on dual-gated bilayer $\mathrm{MoS}_{2}$ devices with metal gates. Direct growth of $\mathrm{HfO}_{2}$ on $\mathrm{MoS}_{2}$ eliminates the indirect bandgap peak and creates artificial PL peak shifts. Also, although we scaled the top gate to $10 \mathrm{~nm}$ gold, it still damped the PL signals severely. For the first problem, we used a HSQ buffer layer to preserve the pristine PL features. To preserve the PL intensity, graphene was used as top gate electrode. Fig. S4 shows the PL measurements after each fabrication steps. After the deposition of a $\mathrm{HSQ}$ buffer layer, $\mathrm{HfO}_{2}$ and graphene, the PL signal loss was less than $15 \%$.

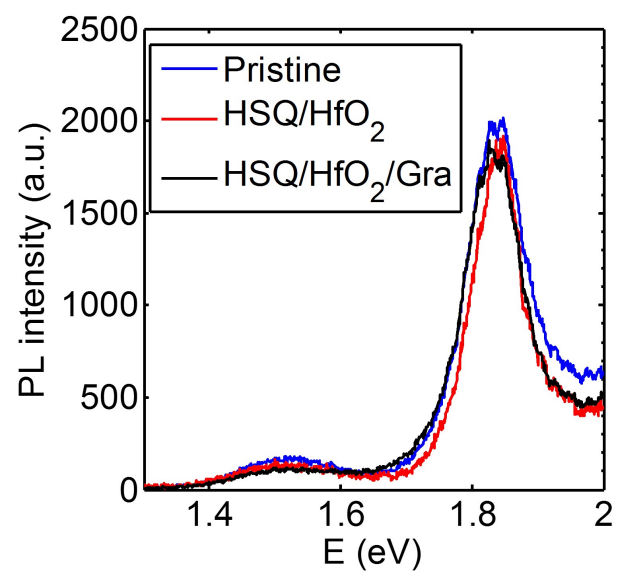

Fig. S4: PL measurements of bilayer MoS2 devices after each fabrications step.

\section{Reproducibility of PL measurements at different electric fields}

To test the reproducibility of the PL response to electric field, three cascading measurements were implemented: (1) $\mathrm{D}_{\mathrm{av}}=0.6 \mathrm{~V} / \mathrm{nm}$, (2) $\mathrm{D}_{\mathrm{av}}=0 \mathrm{~V} / \mathrm{nm}$ and (3) $\mathrm{D}_{\mathrm{av}}=$ $0.6 \mathrm{~V} / \mathrm{nm}$. The PL spectra nicely repeated for measurement 1 and 3 at $\mathrm{D}_{\mathrm{av}}=0.6 \mathrm{~V} / \mathrm{nm}$.

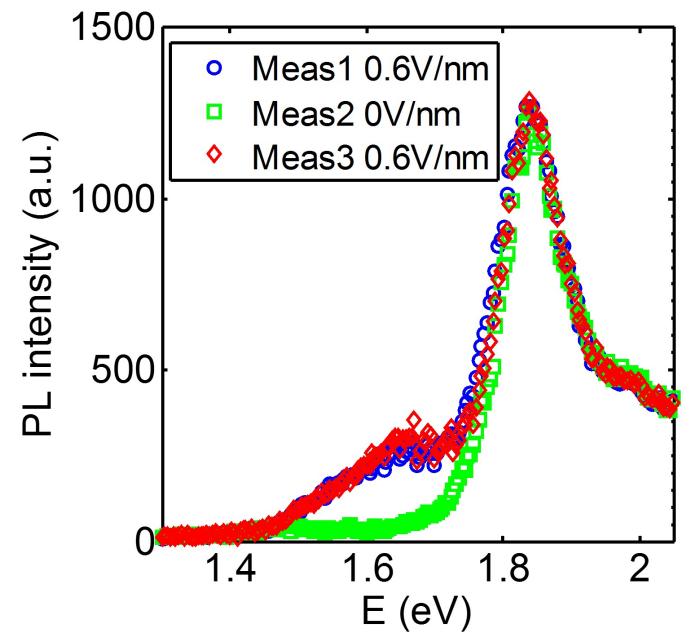

Fig. S5: Reproducibility of PL measurement at different electric fields. 


\section{Semiclassical Approach for PL Prediction}

In the semiclassical theory of light-matter interaction in semiconductors, the radiative recombination rate is proportional to $\mathrm{n}$ times $\mathrm{p}^{\mathrm{S1}}$ :

$\mathrm{PL} \propto \mathrm{np}$

where $\mathrm{n}$ and $\mathrm{p}$ are the electron and hole densities. However, Eq. 1 doesn't consider the wavefunction overlap between electrons and holes. It was shown previously that in the presence of an electric field, the light emission/absorption intensity is proportional to the electron-hole overlap ${ }^{\mathrm{S} 2}$. Without an overlap between $\mathrm{n}$ and $\mathrm{p}$, there is no radiative emission. Hence, Eq. 1 needs to be modified to consider the impact of electron-hole overlap as follows:

$$
\text { PL } \propto \int \mathrm{n}(\mathrm{z}) \mathrm{p}(\mathrm{z}) \mathrm{dz}
$$

Notice that $\mathrm{n}(\mathrm{z})$ and $\mathrm{p}(\mathrm{z})$ are different for transitions I and II (labeled in Fig. 4(c)). For each transition, the density of electrons (holes) is taken from the wavefunction of the corresponding conduction (valence) band where the emission occurs as shown in Fig. S6.

$$
\mathrm{n}(\mathrm{z}) \propto \sum_{\overrightarrow{\mathrm{k}}}\left|\psi_{\mathrm{e}, \overrightarrow{\mathrm{k}}}^{\mathrm{i}}(\mathrm{z})\right|^{2} \mathrm{f}_{\mathrm{e}}, \quad \mathrm{p}(\mathrm{z}) \propto \sum_{\overrightarrow{\mathrm{k}}}\left|\psi_{\mathrm{h}, \overrightarrow{\mathrm{k}}}^{\mathrm{j}}(\mathrm{z})\right|^{2}\left(1-\mathrm{f}_{\mathrm{h}}\right)
$$

where $i$ and $j$ denote the band indices associated with the optical transition of interest, and the functions $f_{e}$ and $f_{h}$ are the electron and hole Fermi-Dirac distribution functions.

\section{Quantum Mechanical Approach for PL Prediction}

Based on Fermi's golden rule, the emission rate for the optical transition depends on the optical matrix element between the conduction and valence band states:

$$
\left.P L\right|_{i \rightarrow j} \propto \sum_{\vec{k}}\left|\left\langle\psi_{h, \vec{k}}^{j}|\vec{r} \cdot \vec{A}| \psi_{e, \vec{k}}^{i}\right\rangle\right|^{2} f_{e}\left(1-f_{h}\right)
$$

where $i$ and $j$ denote the band indices associated with the optical transition of interest. $\overrightarrow{\mathrm{A}}$ is the vector potential of the incident light beam. $\psi_{\mathrm{e}, \overrightarrow{\mathrm{k}}}^{\mathrm{i}}$ and $\psi_{\mathrm{h}, \overrightarrow{\mathrm{k}}}^{\mathrm{j}}$ are the electron and hole wavefunctions with wave-vector $\overrightarrow{\mathrm{k}}$. The k-grid for PL calculation is shown in Fig. S6 (left). 


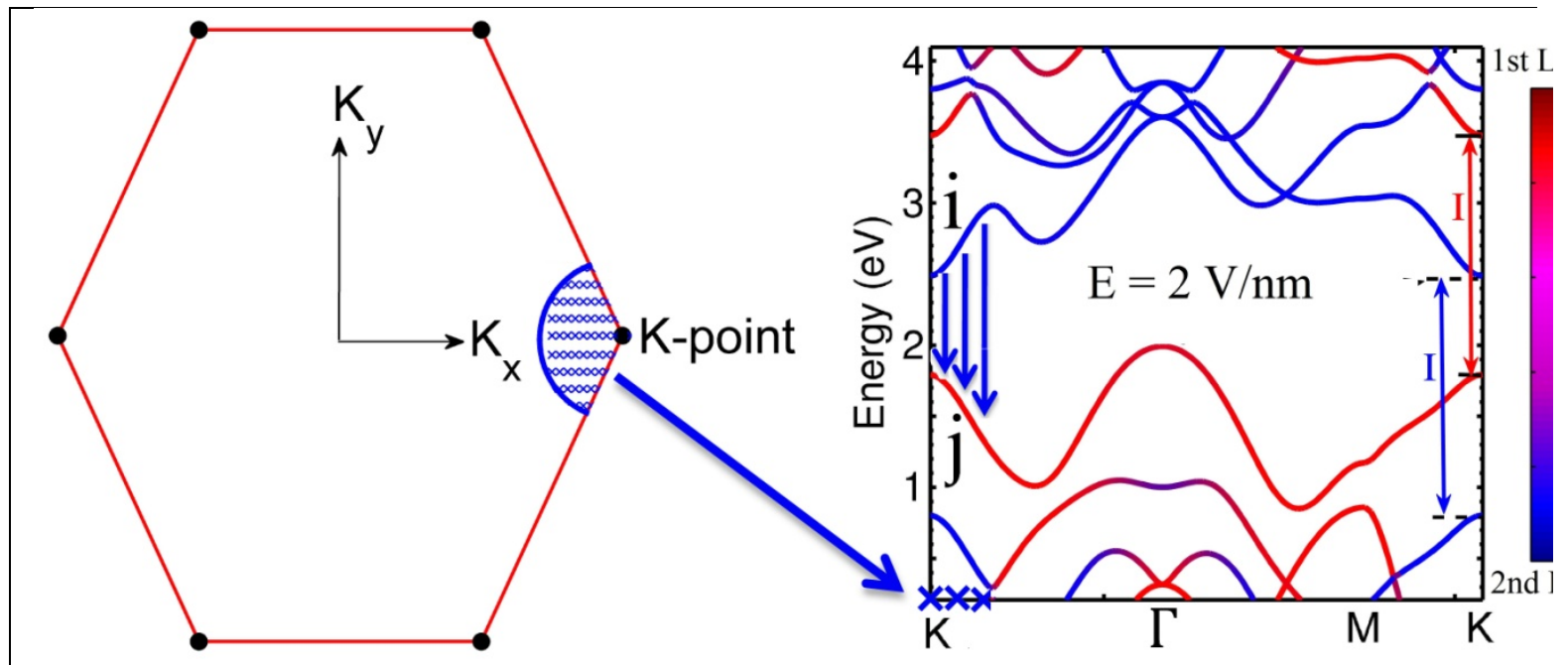

Fig. S6: (Left) Top view of $\mathrm{MoS}_{2}$ Brillouin zone including the k-grid used for the estimation of PL intensity of the transition II. (Right) The energy dispersion showing the transitions I and II.

For each transition, we calculate an effective PL intensity with the emission energy close to $\hbar \omega \approx E^{j}-E^{i}$. To do so, not only the wavefunction overlap between the electron and hole at the band minima and maxima is used, but also the emissions from the states close to the band edges are considered. States with wavevectors far from the K-point in BZ are not considered since they do not contribute to the corresponding transition. These states also have energies much larger than the band minima, and are therefore not occupied (e.g. $f_{e} \propto \exp \left(-\frac{E-E_{c}}{K T}\right)$ ). Hence, these states do not contribute significantly to the PL intensity. To consider the impact of the diminishing carrier population at higher energies, a Boltzmann distribution of carriers in conduction and valence band is considered.

$$
\left.P L\right|_{i \rightarrow j} \propto \sum_{\vec{k}} \mid\left\langle\left.\psi_{h, \vec{k}}^{j}|\vec{r} \cdot \vec{A}| \psi_{e, \vec{k}}^{i}\right|^{2} \exp \left(-\frac{E_{g}^{\vec{k}}-E_{g}^{0}}{K T}\right)\right.
$$

where $\mathrm{E}_{\mathrm{g}}^{\overrightarrow{\mathrm{k}}}$ is the direct bandgap between bands i and $\mathrm{j}$ at wave-vector $\overrightarrow{\mathrm{k}}$ and $\mathrm{E}_{\mathrm{g}}^{0}$ is the direct bandgap at the band minima $\left(\mathrm{E}_{\mathrm{g}}^{0}=\mathrm{E}_{\mathrm{c}}-\mathrm{E}_{\mathrm{v}}\right)$.

Finally, the estimated PL intensity is plotted against the energy of the direct optical transition between the conduction and valence bands $\left(\hbar \omega_{i j} \approx \mathrm{E}^{\mathrm{j}}-\mathrm{E}^{\mathrm{i}}\right)$, as shown in Fig. S7. Notice that only the maximum value of PL is calculated at each electric field. It is well known that the optical emission/absorption has a Lorentzian distribution as a function of the photon energy ${ }^{53}$. Thus a Lorentzian distribution with a half width of $\gamma$ is assumed for the energy dependence of the PL:

$$
\operatorname{PL}(\omega)=\frac{\left.P L\right|_{i \rightarrow j}}{1+\left(\frac{\omega-\omega_{i j}}{\frac{\gamma}{2}}\right)^{2}}
$$


The $\gamma$ value depends on many factors ${ }^{54}$ including the environment (e.g. having the ALD gate dielectric and a graphene top gate). For few layer $\mathrm{MoS}_{2}$ materials, $\gamma$ is normally found to be $\sim 100 \mathrm{meV}$ through fitting ${ }^{55}$. With our specific gate stack, $\gamma$ of $\sim 130 \mathrm{meV}$ is used to fit our experimental curves.

Note that, Fig. 5 (d) from the paper and Fig. S7 are estimates of the PL intensities using two different approaches as discussed above, and the PL values are in arbitrary units. Hence, only the PL intensity ratios as a function of the electric field are of our interest. The quantum mechanical approach (Fig. S7) provides slightly better agreement with the experimental data in terms of the PL peak intensity ratios with different electric fields.

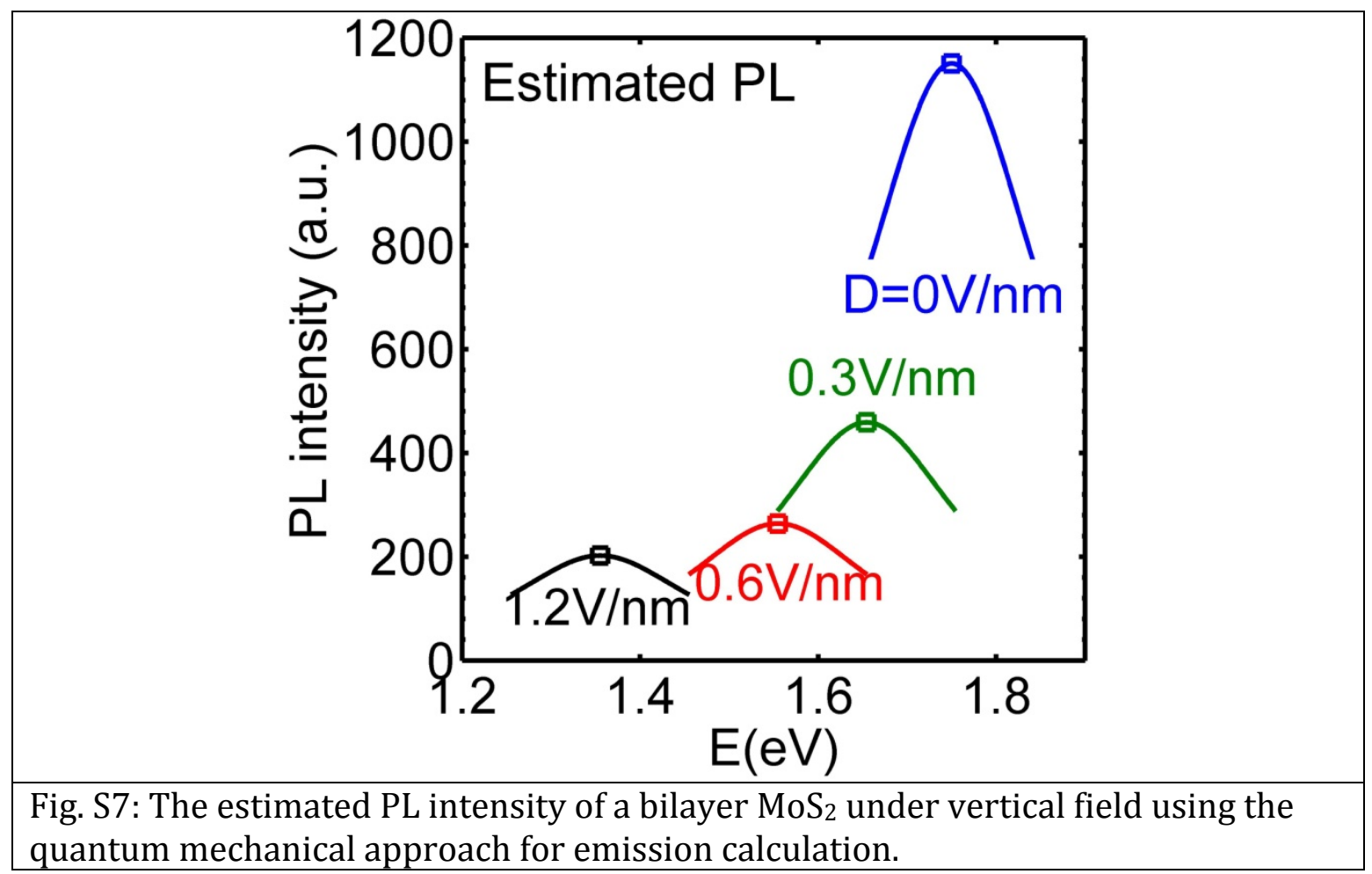

\section{References:}

S1. Desai, S. B. et al. Strain-Induced Indirect to Direct Bandgap Transition in Multilayer WSe 2. Nano Lett. 14, 4592-4597 (2014).

S2. Miller, D., Weiner, J. \& Chemla, D. Electric-field dependence of linear optical properties in quantum well structures: Waveguide electroabsorption and sum rules. IEEE J. Quantum Electron. 22, 1816-1830 (1986). 
S3. Peach, G. Theory of the pressure broadening and shift of spectral lines. Adv. Phys. 30, 367-474 (1981).

S4. Maddams, W. F. The Scope and Limitations of Curve Fitting. Appl. Spectrosc. 34, 245-267 (1980).

S5. Sercombe, D. et al. Optical investigation of the natural electron doping in thin MoS2 films deposited on dielectric substrates. Sci. Rep. 3, 3489 (2013). 